\title{
Validation of the Korean Ford Insomnia Response to Stress Test Questionnaire
}

\author{
Jinyoung Chang, BA, Sooyeon Suh, PhD \\ Department of Psychology, Sungshin Women's University, Seoul, Korea
}

Received: December 3, 2018 Accepted: December 20, 2018

Correspondence

Sooyeon Suh, PhD

Department of Psychology,

Sungshin Women's University,

2 Bomun-ro 34-da gil, Seongbuk-gu,

Seoul 02844, Korea

Tel $+82-2-920-7215$

Fax +82-2-920-2040

E-mail alysuh@sungshin.ac.kr

ORCID

Jinyoung Chang

https://orcid.org/0000-0002-0441-0061

Sooyeon Suh

https://orcid.org/0000-0003-0644-8634
Background and Objective This study aimed to examine psychometric properties of the Korean Ford Insomnia Response to Stress Test (K-FIRST). The FIRST measures personal vulnerability of sleep difficulties in response to stressful events.

Methods 377 participants (mean age $=21.80 \pm 2.91$ years, $76.9 \%$ female) completed online surveys about demographic information, sleep reactivity (K-FIRST), insomnia (Insomnia Severity Index, ISI), perceived stress (Perceived Stress Scale, PSS), and depression (Center for Epidemiological Studies-Depression Scale, CES-D). Exploratory factor analysis was conducted to determine the factor structure of the K-FIRST.

Results K-FIRST showed adequate internal consistency (Cronbach's $\alpha=0.85$ ) and significant goodness of fit test result $\left(\chi^{2}=1386.33, p<0.001\right)$. A two-factor structure was selected as a result of the factor analysis, with rumination and worry as the two factors. K-FIRST was significantly associated with the ISI $(\mathrm{r}=0.35, \mathrm{p}<0.01)$, PSS $(\mathrm{r}=0.30, \mathrm{p}<0.01)$, and CES-D $(\mathrm{r}=0.42, \mathrm{p}<0.01)$.

Conclusions The results of this present study supported the reliability and validity of K-FIRST. It is expected that the K-FIRST can be used in future research in investigating personal vulnerabilities that may predispose individuals to sleep disturbance.

Sleep Med Res 2018;9(2):92-96

Key Words Sleep difficulties, Insomnia, Sleep reactivity, Validation, Worry and rumination.

\section{INTRODUCTION}

The prevalence of insomnia has been increasing in Korea, with more than one of five Koreans reporting insomnia symptoms [1]. There are many theories and models that explain onset and maintenance of insomnia. According to the 3-P model of insomnia, there are predisposing, precipitating, and perpetuating factors [2]. Precipitating factors trigger sleep disturbance or acute insomnia that could be a stressful life event. However, everyone who experiences stress does not experience sleep disturbance. This difference could partially be explained by predisposing factors, which can be defined as personal vulnerabilities that are present prior to the onset of insomnia [2,3].

Sleep reactivity is defined as a vulnerability that increases the likelihood of sleep disturbance when exposed to variety of stressful situations, and it has been reported to be influenced by genetic and environmental factors [4,5]. Drake and his colleagues [6] have found that individuals with high sleep reactivity reported higher levels of sleep disturbance following stressful events compared to those with low sleep reactivity.

The Ford Insomnia Response to Stress Test (FIRST) is a self-report measure developed to measure sleep reactivity, including items that assess the extent that one suffers from sleep difficulty following stressful life events [4]. The FIRST has been used in various studies with many clinical populations, such as shift workers, elders, and young adults, and also in various countries $[4,6-12]$. 
The current study aimed to validate the Korean version of the FIRST (K-FIRST) by investigating the internal reliability, factor structure, and convergent and discriminant validity. Also, this study also investigated associations of sleep reactivity with insomnia severity, depression, and stress level dependence on high and low sleep reactivity.

\section{METHODS}

\section{Participants and Settings}

Participants for the study were recruited through classroom announcements and online advertisements between 2016 and 2017. A total of 384 consenting participants completed online self-report questionnaires. The Institutional Review Board at Sungshin Women's University approved the study (SSWUIRB 2016-027, SSWUIRB 2016-063, and SSWUIRB 2017-016) and all participants signed a consent form before participating in the study.

\section{Measures}

FIRST: FIRST is a self-report instrument assessing vulnerability to sleep disturbances in response to nine stressful life events [4]. All items are rated on a 4-point Likert scale ranging from "not likely (1)" and "very likely (4)." The total score ranges from 9 to 36, with higher scores indicating greater vulnerability to sleep disturbances. For the current study, the original version of FIRST was translated and back translated in Korean by two independent individuals who were bilingual.

Insomnia Severity Index (ISI): The ISI is a 7-item self-report measurement that assesses insomnia severity asking about perceived insomnia severity and functional impairment in the past two weeks [13]. The ISI uses items rated on a 4-point Likert scale ranging from 0 to 4 , with total scores ranging from 0 to 28. Higher scores reflect more severe symptoms of insomnia. The present study used ISI in Korean, which was translated by Cho et al. [14].

Perceived Stress Scale (PSS): The PSS measures perceived stress levels in the past one month [15]. Each 10 items respond to a 5-point Likert scale, with higher scores reflecting higher perceived stress. The total score ranges from 0 to 40 . The PSS translated by Lee and Lee [16] was used in this study.

Center for Epidemiologic Studies Depression Scale (CES-D): The CES-D is a self-report questionnaire that measures behaviors and cognitions associated with depression in the past week [17]. The questionnaire consists of 20 items, with items scored on a 4-point Likert scale. Total scores ranges from 0 to 60 ; higher scores indicates higher depression level. This study used a Korean version of CES-D, which was validated and revised by Chon et al. [18].

\section{Data Analysis}

Data was analyzed using SPSS 21.0 (IBM Corp., Armonk, NY, USA). Factor analysis was conducted by Mplus 7.11 (Muthen \& Muthen, Los Angeles, CA, USA). First, descriptive statistics using mean, standard deviation and frequency analyses were conducted to analyze participant characteristics.

Cronbach's a was used to calculate internal consistency. Additionally, correlation coefficients were calculated between KFIRST, ISI, PSS, and CES-D. T-tests were used to compare the high and low sleep reactivity groups. The cut-off score of FIRST was 16 divided into groups [19]. Before performing factor analysis, sampling adequacy was verified using Bartlett spherical test and Kaiser-Meyer-Olkin test (KMO). Exploratory factor analysis (EFA) with maximum likelihood was used, and factors were rotated using Geomin. Geomin is one of the oblique rotations that minimizes variable complexity $[20,21]$. To assess adequate fit of data for EFA, this study followed the following standard recommendations. If the Comparative Fit Index and Tucker Lewis Index values are above 0.95 , it is considered an excellent adequate fit. Less than 0.08 of root mean square error of approximation and $\leq 0.10$ of standardized root mean square residual presents a good fit $[22,23]$

\section{RESULTS}

\section{Demographic and Clinical Information}

377 participants (mean age $=21.80 \pm 2.91$ years, $76.9 \% \mathrm{fe}$ male) completed the questionnaire. The majority of participants were students. Demographic information is presented in Table 1.

Participants were divided into two groups (high and low sleep reactivity) based on their FIRST score, using a cut-off score of 16 [19]. Among the sample, 259 participants $(21.49 \pm 2.80$ years, $82.2 \%$ female) were classified as the high sleep reactivity group, and 118 participants were classified as the low sleep reactivity group ( $22.49 \pm 3.04$ years, $65.3 \%$ female). Participants in the high sleep reactivity group had significantly higher scores on the ISI, PSS, and CES-D compared to the low sleep reactivity group $(\mathrm{p}<0.001)$ (Table 1$)$.

\section{Reliability of the FIRST}

The internal consistency of the FIRST was adequate (Cronbach's $\alpha=0.85$ ), indicating that the scale was homogenous. All items correlated significantly with the full scale (range 0.22$0.80, \mathrm{p}<0.01$ ) (Table 2). Also, the self-reported instrument of ISI, PSS, and CES-D's Cronbach's $\alpha$ range was $0.60-0.80$ (Table 3 ).

\section{Validity of the FIRST}

The Bartlett spherical test was significant $\left(\chi^{2}=1386.33, \mathrm{p}<\right.$ 0.001 ) and the KMO was 0.87 . According to these results, factor analysis was determined to be appropriate. EFA was conducted using maximum likelihood estimation. According to 
Table 1. Characteristics of sample $(n=377)$

\begin{tabular}{lcccc}
\hline & Total & High FIRST & Low FIRST & p \\
\hline Demographic & & & & \\
Age (yr), $\mathrm{n}=373$ & $21.80 \pm 2.91$ & $21.49 \pm 2.80$ & $22.49 \pm 3.04$ & $0.002^{*}$ \\
$\quad$ Sex (women, \%) & 76.90 & 82.20 & 65.30 & $0.001^{*}$ \\
Measure & & & & $<0.000^{\dagger}$ \\
FIRST & $19.16 \pm 6.20$ & $22.25 \pm 4.85$ & $12.36 \pm 1.89$ & $<0.000^{\dagger}$ \\
ISI & $9.40 \pm 4.86$ & $10.20 \pm 4.98$ & $7.64 \pm 4.07$ & $<0.000^{\dagger}$ \\
PSS & $18.19 \pm 4.91$ & $19.06 \pm 4.74$ & $16.29 \pm 4.73$ & $<0.000^{\dagger}$ \\
CES-D & $17.20 \pm 10.32$ & $19.25 \pm 10.82$ & $12.69 \pm 7.40$ & \\
\hline
\end{tabular}

${ }^{*} \mathrm{p}<0.01 .{ }^{\dagger} \mathrm{p}<0.001$.

FIRST: Ford Insomnia Response to Stress Test, ISI: Insomnia Severity Index, PSS: Perceived Stress Scale, CES-D: Center for Epidemiologic Studies Depression Scale.

Table 2. Correlation between FIRST items

\begin{tabular}{|c|c|c|c|c|c|c|c|c|c|}
\hline & 1 & 2 & 3 & 4 & 5 & 6 & 7 & 8 & 9 \\
\hline FIRST 1 & 1 & & & & & & & & \\
\hline FIRST 2 & $0.45^{*}$ & 1 & & & & & & & \\
\hline FIRST 3 & $0.46^{*}$ & $0.80^{*}$ & 1 & & & & & & \\
\hline FIRST 4 & $0.50^{*}$ & $0.68^{*}$ & $0.65^{*}$ & 1 & & & & & \\
\hline FIRST 5 & $0.29^{*}$ & $0.25^{*}$ & $0.26^{*}$ & $0.28^{*}$ & 1 & & & & \\
\hline FIRST 6 & $0.32 *$ & $0.56^{*}$ & $0.52^{*}$ & $0.48^{*}$ & $0.24^{*}$ & 1 & & & \\
\hline FIRST 7 & $0.33^{*}$ & $0.45^{*}$ & $0.52^{*}$ & $0.48^{*}$ & $0.26^{*}$ & $0.38^{*}$ & 1 & & \\
\hline FIRST 8 & $0.43^{*}$ & $0.30^{*}$ & $0.29 *$ & $0.36^{*}$ & $0.26^{*}$ & $0.32 *$ & $0.31^{*}$ & 1 & \\
\hline FIRST 9 & $0.50^{*}$ & $0.22 *$ & $0.29^{*}$ & $0.33^{*}$ & $0.27^{*}$ & $0.25^{*}$ & $0.36^{*}$ & $0.46^{*}$ & 1 \\
\hline
\end{tabular}

${ }^{*} \mathrm{p}<0.01$.

FIRST: Ford Insomnia Response to Stress Test.

the scree plot and eigenvalue, the Korean version of the FIRST, suggested single- or two-factor model. The two-factor model demonstrated better goodness-of-fit indices compared to the one-factor model (Table 4). Based on goodness-of-fit indices, the K-FIRST adopted a 2-factor structure in which all items showed factor loading estimates after Geomin rotation were 0.4 or greater except on item Q5.

In this case, Q5 should be deleted from the FIRST; however, Q5 was kept to compare the total score time with the original FIRST. Items Q2-Q4 and Q6-Q7 were loaded on the first factor, whereas items Q1 and Q8-Q9 were loaded on the second factor (Table 5).

The correlation between factor 1 and 2 was 0.43 , indicating a statistically significant yet independent relationship since the correlation did not exceed 0.85 (Table 6) [24,25].

\section{Association of the K-FIRST with Other Measures}

There were significant correlations between the K-FIRST with ISI, PSS, and CES-D measures $(\mathrm{p}<0.001)$. High K-FIRST group showed higher scores of ISI, PSS, and CES-D than low K-FIRST.
Table 3. Correlation between instruments

\begin{tabular}{lcccc}
\hline & FIRST & ISI & PSS & CES-D \\
\hline FIRST & 1 & & & \\
ISI & $0.35^{*}$ & 1 & & \\
PSS & $0.30^{*}$ & $0.34^{*}$ & 1 & \\
CES-D & $0.42^{*}$ & $0.43^{*}$ & $0.70^{*}$ & 1 \\
\hline
\end{tabular}

${ }^{*} \mathrm{p}<0.01$.

FIRST: Ford Insomnia Response to Stress Test, ISI: Insomnia Severity Index, PSS: Perceived Stress Scale, CES-D: Center for Epidemiologic Studies Depression Scale.

\section{DISCUSSION}

The current study aimed to validate the Korean version of the FIRST. This study validated the FIRST in a Korean sample of young adults and investigated the associations between KFIRST and insomnia severity, perceived stress, and depression.

In this study, K-FIRST demonstrated adequate internal consistency, and reliability data was similar to other validation studies of the FIRST. EFA results also indicated that K-FIRST supported a two-factor model compared to a single factor model 
Table 4. Goodness-of-fit indices for exploratory factor model of FIRST

\begin{tabular}{lcccccc}
\hline & $\chi^{2}(\mathrm{df})$ & $\mathrm{p}$ & CFI & TLI & RMSEA & SRMR \\
\hline 1 factor & $215.68(27)$ & $<0.000^{*}$ & 0.86 & 0.82 & 0.14 & 0.08 \\
2 factor & $29.81(19)$ & $>0.05$ & 0.99 & 0.99 & 0.04 & 0.02 \\
\hline
\end{tabular}

${ }^{*} \mathrm{p}<0.001$.

FIRST: Ford Insomnia Response to Stress Test, CFI: Comparative Fit Index, TLI: Tucker Lewis Index, RMSEA: root mean square error of approximation, SRMR: standardized root mean square residual.

Table 5. Factor loading estimates after Geomin rotation

\begin{tabular}{lcc}
\hline & 1-factor & 2-factor \\
\hline $\begin{array}{l}\text { Q1. Before an important meeting the next day } \\
\text { Q2. After a stressful experience during the day }\end{array}$ & $0.97^{*}$ & \\
Q3. After a stressful experience in the evening & $0.87^{*}$ & \\
Q4. After getting bad news during the day & $0.69^{*}$ & \\
Q5. After watching a frightening movie or TV & & $0.30^{*}$ \\
show & $0.57^{*}$ & \\
Q6. After having a bad day at work & $0.44^{*}$ & \\
Q7. After an argument & & $0.55^{*}$ \\
Q8. Before having to speak in public & $0.77^{*}$ \\
Q9. Before going on vacation the next day &
\end{tabular}
*p $<0.05$.

Table 6. Factor correlation between 1-factor and 2-factor model

\begin{tabular}{lcc}
\hline & 1 factor & 2 factor \\
\hline 1 -factor & 1 & \\
2 -factor & $0.43^{*}$ & 1 \\
${ }^{*} \mathrm{p}<0.05$. & &
\end{tabular}

for the K-FIRST. Since the FIRST was developed, it has been validated in various countries such as Japan, Italy, and Portugal etc. $[4,10,12,26]$. The factor structure of the FIRST has varied across countries. While the original FIRST yielded a 1-factor model, subsequent validations of the FIRST have found the FIRST to support a multi-factor model. While the Japanese version of the FIRST supported the 1-factor model similar to the original version, the Portuguese version reported a 2-factor model (rumination and worry); the Italian version of FIRST extracted three components that metacognitive beliefs could be related to sleep reactivity $[10,12,26]$. The reasons for differences in various factor structures are unclear, but likely due to heterogeneous methodology and cross-cultural factors. For the K-FIRST, the items loaded on similar factors as the Portuguese version. In particular, item Q5 did not load in both Korean and Portuguese versions of FIRST, likely due to both samples being mainly college students [12]. Unlike the Korean and Portuguese studies, the original and Japanese version participants mean ages were mainly middle-aged $[4,10]$. Additionally, according to Taylor and his colleagues [27], cultural difference could have also affected the results.

Worry and rumination were distinguished from K-FIRST items as a two-factor model. The K-FIRST validated as two- factor model in this study and it was similar outcome to the Portuguese version of the FIRST, which also yielded a two-factor model, labeled rumination and worry (worry = Q1, Q8, and Q9; rumination = Q2, Q3, Q4, Q6, and Q7). Rumination is related to thinking about stressful events after they have happened. In comparison, worry activates before experiencing a stressful event. The concepts of rumination and worry, while related, are usually viewed as independent constructs and considered as part of repetitive thought process [28]. Maladaptive metacognitions about repetitive thought is one of mechanisms to maintain repetitive thoughts that are related to both rumination and worry [29,30]. Sleep disturbance is related to cognitive arousal that is explained by repetitive thoughts, and rumination and worry are often present that prolongs sleep onset latency [30,31]. Several studies which informed participants that they had to give a speech upon waking before they go to bed increased worry in participants, which led to sleep disturbances [30,32-34]. Thus, worry is often associated with future events. In contrast, there was a study showed people with higher trait rumination experienced more sleep disturbance than lower people, and frequently thought content is associated with rethinking past events $[30,35]$. Findings from validation studies of FIRST were supported by previous studies. Based on past studies, the FIRST score was significantly correlated with rumination and closely related with metacognitive beliefs about sleep.

This study divided participants into high and low sleep reactivity groups, similar to previous studies $[4,10,12]$. Results indicated that high sleep reactivity group reported higher insomnia severity, perceived stress, and depression than low sleep reactivity group. These results are consistent with previous studies and reflect that people with high sleep reactivity may frequently experience severe sleep disturbance, more stress, and undergo greater depression symptoms.

\section{Limitations}

This study has several limitations. First, only self-report questionnaires were used measuring insomnia and other clinical indices. Thus, it is necessary to use objective sleep measures like actigraphy in future studies. Second, the results could be difficult to generalize due to the homogeneous nature of the sample. In this study, the proportion of women was high. Finally, the present study did not test test-retest validity, and thus may have been difficult to ascertain sleep reactivity as a personal trait compared to state in this study. 


\section{Conclusion}

In conclusion, this study aimed to establish the psychometric properties of FIRST in a sample of Korean population. Our findings showed that the K-FIRST demonstrated good reliability and validity. The K-FIRST was validated as two-factor model unlike the original version, with rumination and worry as the two prevailing factors. It is expected that the K-FIRST can be used in future research in investigating personal vulnerabilities that may predispose individuals to sleep disturbance.

\section{Acknowledgments}

This work was supported by Sungshin University Research Grant of 2017 (2017-1-29-007).

\section{Conflicts of Interest}

The authors have no financial conflicts of interest.

\section{Authors' Contribution}

Conceptualization: Chang J, Suh S. Data curation: Chang J, Suh S. Formal analysis: Chang J. Methodology: Chang J, Suh S. Writing_original draft: Chang J. Writing_review \& editing: Suh S.

\section{REFERENCES}

1. Cho YW, Shin WC, Yun CH, Hong SB, Kim J, Earley CJ. Epidemiology of insomnia in Korean adults: prevalence and associated factors. J Clin Neurol 2009;5:20-3.

2. Spielman AJ, Caruso LS, Glovinsky PB. A behavioral perspective on insomnia treatment. Psychiatr Clin North Am 1987;10:541-53.

3. Perlis M, Shaw PJ, Cano G, Espie CA. Models of insomnia. In: Kryger $\mathrm{MH}$, Roth T, Dement WC. Principles and practice of sleep medicine. 5th ed. Philadelphia: Elsevier Saunders 2011;850-65.

4. Drake C, Richardson G, Roehrs T, Scofield H, Roth T. Vulnerability to stress-related sleep disturbance and hyperarousal. Sleep 2004;27:28591.

5. Bonnet MH, Arand DL. Situational insomnia: consistency, predictors, and outcomes. Sleep 2003;26:1029-36.

6. Drake CL, Friedman NP, Wright KP Jr, Roth T. Sleep reactivity and insomnia: genetic and environmental influences. Sleep 2011;34:1179-88.

7. Fernández-Mendoza J, Vela-Bueno A, Vgontzas AN, Ramos-Platón MJ, Olavarrieta-Bernardino S, Bixler EO, et al. Cognitive-emotional hyperarousal as a premorbid characteristic of individuals vulnerable to insomnia. Psychosom Med 2010;72:397-403.

8. Kalmbach DA, Pillai V, Cheng P, Arnedt JT, Drake CL. Shift work disorder, depression, and anxiety in the transition to rotating shifts: the role of sleep reactivity. Sleep Med 2015;16:1532-8.

9. Lim YC, Kim SJ, Kwon HJ, Lee JH. The effects of stress- and sleep-related variables on the quality of life in insomnia patients. J Korean Neuropsychiatr Assoc 2016;55:277-85.

10. Nakajima S, Okajima I, Sasai T, Kobayashi M, Furudate N, Drake CL, et al. Validation of the Japanese version of the Ford Insomnia Response to Stress Test and the association of sleep reactivity with trait anxiety and insomnia. Sleep Med 2014;15:196-202.

11. Palagini L, Faraguna U, Mauri M, Gronchi A, Morin CM, Riemann D. Association between stress-related sleep reactivity and cognitive processes in insomnia disorder and insomnia subgroups: preliminary results. Sleep Med 2016;19:101-7.

12. Marques DR, Allen Gomes AC, Drake CL, Roth T, de Azevedo MHP. Assessing stress-induced sleep reactivity in college students: The European Portuguese version of the Ford Insomnia Response to Stress Test
(FIRST). Behav Sleep Med 2018;16:337-46.

13. Bastien $\mathrm{CH}$, Vallières A, Morin CM. Validation of the Insomnia Severity Index as an outcome measure for insomnia research. Sleep Med 2001;2:297-307.

14. Cho YW, Song ML, Morin CM. Validation of a Korean version of the insomnia severity index. J Clin Neurol 2014;10:210-5.

15. Cohen S, Kamarck T, Mermelstein R. A global measure of perceived stress. J Health Soc Behav 1983;24:385-96.

16. Lee J, Lee M. The effects of self-complexity and self-efficacy on depression and perceived stress [master's thesis]. Suwon, Korea: Aju University 2005.

17. Radloff LS. The CES-D scale: a self-report depression scale for research in the general population. Appl Psychol Meas 1977;1:385-401.

18. Chon KK, Choi SC, Yang BC. Integrated adaptation of CES-D in Korea. Korean J Health Psychol 2001;6:59-76.

19. Kalmbach DA, Pillai V, Arnedt JT, Drake CL. Identifying at-risk individuals for insomnia using the Ford Insomnia Response to Stress Test. Sleep 2016;39:449-56.

20. Thurstone LL. Multiple factor analysis: a development and expansion of vectors of the mind. Chicago: University of Chicago Press 1947.

21. Sass DA, Schmitt TA. A comparative investigation of rotation criteria within exploratory factor analysis. Multivariate Behav Res 2010;45:73103.

22. Hu L, Bentler PM. Cutoff criteria for fit indexes in covariance structure analysis: conventional criteria versus new alternatives. Struct Equ Modeling 1999;6:1-55.

23. Browne MW, Cudeck R. Alternative ways of assessing model fit. Sage Focus Editions 1993;154:136-36.

24. Kline R. Principles and practice of structural equation modeling. New York: Guilford Press 1998.

25. Abdullah NJS, Ismail IA, Idrus K, Krauss SE, Abdullah AL. Relationship between organizational antecedent and knowledge sharing practices among academician at Malaysia Research Universities. Sains $\mathrm{Hu}$ manika 2015;5:99-108.

26. Palagini L, Bruno RM, Paolo T, Caccavale L, Gronchi A, Mauri M, et al. Association between stress-related sleep reactivity and metacognitive beliefs about sleep in insomnia disorder: preliminary results. Behav Sleep Med 2016;14:636-49.

27. Taylor GJ, Bagby RM, Parker JD. The 20-Item Toronto Alexithymia Scale. IV. Reliability and factorial validity in different languages and cultures. J Psychosom Res 2003;55:277-83.

28. Carney CE, Harris AL, Moss TG, Edinger JD. Distinguishing rumination from worry in clinical insomnia. Behav Res Ther 2010;48:540-6.

29. Clark DA. Unwanted mental intrusions in clinical disorders: an introduction. J Cogn Psychotherapy 2002;16:123-6.

30. Pillai V, Drake CL. Sleep and repetitive thought: the role of rumination and worry in sleep disturbance. In: Babson KA, Feldner MT. Sleep and affect. Academic Press 2015;201-25.

31. Harvey AG. Unwanted intrusive thoughts in insomnia. In: Clark DA. Intrusive thoughts in clinical disorders: Theory, research, and treatment. New York: Guilford Press 2005;86-118.

32. Hall M, Buysse D, Reynolds C, Kupfer D, Baum A. Stress-related intrusive thoughts disrupt sleep onset and continuity. Sleep Res 1996;25:16374 .

33. Gross RT, Borkovec TD. Effects of a cognitive intrusion manipulation on the sleep-onset latency of good sleepers. Behavior Therapy 1982;13: $112-6$.

34. Tang NK, Harvey AG. Effects of cognitive arousal and physiological arousal on sleep perception. Sleep 2004;27:69-78.

35. Thomsen DK, Mehlsen MY, Christensen S, Zachariae R. Rumination-relationship with negative mood and sleep quality. Pers Indiv Differ 2003;34:1293-301. 